\title{
A Petrov-Galerkin Finite Element Method for Solving the Time-fractional Diffusion Equation with Interface
}

\author{
Liwei Shi ${ }^{1}$ \\ ${ }^{1}$ Department of Science and Technology, China University of Political Science and Law, Beijing, China \\ Correspondence: Liwei Shi, Department of Science and Technology, China University of Political Science and Law, \\ Beijing, China. E-mail: shiliweita@126.com
}

Received: June 13, 2018 Accepted: July 5, 2018 Online Published: July 25, 2018

doi:10.5539/jmr.v10n4p136 URL: https://doi.org/10.5539/jmr.v10n4p136

The research is financed by Science Foundation of China University of Political Science and Law (No.16ZFQ11001).

\begin{abstract}
Time-fractional partial differential equation is widely applied in a variety of disciplines, its numerical solution has attracted much attention from researchers in recent years. Time-fractional differential equations with interfaces is a more challenging problem because the governing equation has discontinuous coefficients at interfaces and sometimes singular source term exists. In this paper, we propose a Petrov-Galerkin finite element method for solving the two-dimensional time-fractional diffusion equation with interfaces. In this method, a finite difference scheme is employed in time and a Petrov-Galerkin finite element method is employed in space. Extensive numerical experiments show that for a fractional diffusion equation of order $\alpha$ with interfaces, our method gets to $(2-\alpha)$-order accurate in the $L^{2}$ and $L^{\infty}$ norm.
\end{abstract}

Keywords: time-fractional partial differential equation, sharp-edged interface, Petrov-Galerkin finite element method, finite difference method

\section{Introduction}

Fractional differential equations are generalized differential operations from integer orders to fractional derivative operations. The problem of fractional calculus has wide applications in fractal theory, signal processing, system control, quantum mechanics, environmental science, and finance (Jiang and Qi, 2010; Mainardi, 1996). The time-fractional differential equation is obtained by replacing the integer order in the classical model to fractional derivative, the fractional derivative at a certain time depends on all the values of the function before this time point, so the fractional partial differential equation is applicable for problems with memory process, genetic property and heterogeneous material (Rossikhin and Shitikova, 1997; Ichise et al., 1971). Laplace and Fourier transform are employed to get the analytical solutions of some fractional partial differential equations, for example, equations with constant coefficients (Baeumer et al., 2005; Duan, 2005; Meerschaert et al., 2002). But for most fractional partial differential equations, analytical solutions are not available, in recent decades, the numerical solution of fractional differential equations has attracted much attention from researchers, numerical methods that are employed include finite difference method (Langlands and Henry, 2005; Liu et al., 2004; Meerschaert and Tadjeran, 2006; Su et al., 2009; Murio, 2008), finite element method (Ervin et al., 2007; Deng, 2008; Burrage et al., 2012; Jiang and Ma, 2011), discontinuous Galerkin method (Mustapha and McLean, 2011; Ji and Tang, 2012), spectral method (Zayernouri and Karniadakis, 2014; Zheng and Wei, 2010), meshless method (Liu et al., 2011; Dehghan et al., 2015), and some other methods (Ray, 2007; El-Wakil et al., 2009; Rihan, 2010).

In (Liu et al., 2002, 2003; Huang and Liu, 2005), Liu et al. derived the numerical methods for solving the fractional advection-dispersion equation. In (Liu et al., 2007), the stability and convergence of the difference methods are studied. Meerschaert et al. (Meerschaert et al., 2006) used a variation of the Grünwald finite difference approximation to solve two-dimensional fractional partial differential equations with variable coefficients. Roop (Roop, 2006) pointed out that the main applying the finite element method to the numerical solution of fractional partial differential equations is that the fractional operater is non-local, the resulting matrix is almost dense, so it needs higher computational costs. Zeng et al. (Zeng et al., 2015) provided a second-order accurate scheme for the time-fractional subdiffusion equation. The authors gave strict analysis of the stability and convergence of this method. Compared with the finite difference methods and finite element methods, the meshless method has the advantage that they use higher order trial functions, so that they usually have higher accuracy. (Liu et al., 2011) used an implicit meshless radial basis function(RBF) to solve the time fractional diffusion equations. Dehghan et al. (Dehghan et al., 2015) used the meshless method to solve the time fractional sine-Gordon and Klein-Gordon equations. In ( $\mathrm{Li}$ and Xu, 2009), a spectral method is proposed to solve the time fractional diffusion equation. The authors formulated the time fractional fractional differential problem into an elliptic problem by finding proper spaces and norms. Zheng et al. (Zheng et al., 2015) presented a space-time spectral method for the time 
fractional Fokker-Planck equation with high order accuracy and efficiency.

In order to simulate problems with irregular convex domains, Fan et al. (Fan et al., 2017) proposed an unstructured mesh finite element method to solve the time-space fractional wave equation on an irregular convex domain. For many practical problems, interfaces are involved in the research domain. Interface problems attract much interest in recent years because of their wide applications in fluid dynamics, electromagnetic, biological systems (Chadam and Yin, 1993; Kandilarov, 2005), etc. The pioneering work was done by Peskin in the simulation of blood flow in heart. He proposed the "immersed boundary" method (Peskin, 1977; Peskin and Printz, 1993). In (Sussman et al., 1994), the "immersed boundary" method was combined with the level set method, resulting in a first order numerical method that is simple to implement, even in multiple spatial dimensions. The "immersed interface" method presented in (LeVeque and Li, 1994) can get second-order accuracy. (Chen and Zou, 1998) used finite element method to solve elliptic problems with the interface conditions $[u]=0$ and $\left[\beta u_{n}\right] \neq 0$, second order accuracy in the energy norm and nearly second order accuracy in the $L^{2}$ norm can be achieved. The boundary condition capturing method (Liu et al., 2000) uses the Ghost fluid method (GFM) (Fedkiw et al., 1999) to capture the boundary conditions. This method was sped up by a multi-grid method (Justin and Liu, 2004), and in(Macklin and Lowengrub, 2008), the method is improved to second order accuracy for smooth interfaces. In (Zhou et al., 2006), the matched interface and boundary (MIB) method was proposed to solve elliptic equations with smooth interfaces. In (Yu et al., 2007), the MIB method was generalized to treat sharp-edged interfaces. (Colella and Johansen, 1998; Oevermann and Klein, 2006; Oevermann et al., 2009) developed high order methods for elliptic equations in complex domains from the finite volume perspective. (Ying and Henriquez, 2007) is a kernel-free boundary integral (KFBI) method for solving elliptic BVPs. In (Hou and Liu, 2005), a finite element method with non-body-fitting grids is proposed to solve elliptic equations with matrix coefficients and sharp-edged interfaces. In (Wang et al., 2017, 2018), the method is improved to solve three-dimensional problems.

Although the numerical solution of fractional differential equations and interface problems have gained much attention respectively, there are few research about the fractional differential equations with interfaces. The main challenge of solving this kind of problem is that the solution at current time level, the coefficient matrix and the jump conditions all depend on the solutions at all previous time levels because of the existence of interfaces and fractional derivative in time. In this paper, we propose a Petrov-Galerkin finite element method to solve the time-fractional diffusion equation with interfaces. The main idea is to use a finite difference scheme in time and a Petrov-Galerkin finite element method with non-body-fitting grids in space. Extensive numerical experiments demonstrate the effectiveness of our method.

\section{Equations and Weak Formulation}

Consider an open bounded domain $\Omega \subset R^{d}$. We assume that there is a Lipschitz continuous and piecewise smooth level-set function $\phi$ on $\Omega$. Let $\Gamma=\{\phi=0\}$ be an interface of co-dimension $d-1$, which divides $\Omega$ into disjoint open subdomains, $\Omega^{-}=\{\phi<0\}$ and $\Omega^{+}=\{\phi>0\}$, hence $\Omega=\Omega^{-} \cup \Omega^{+} \cup \Gamma$. Assume that the boundary $\partial \Omega$ and the boundary of each subdomain $\partial \Omega^{ \pm}$are Lipschitz continuous as submanifolds. Since $\partial \Omega^{ \pm}$are Lipschitz continuous, so is $\Gamma$. A unit vector $\boldsymbol{n}=\left[\begin{array}{l}n_{x} \\ n_{y}\end{array}\right]=\frac{\nabla \phi}{|\nabla \phi|}$ can be obtained on $\bar{\Omega}$, which is a unit normal vector of $\Gamma$ pointing from $\Omega^{-}$to $\Omega^{+}$.

Let $T>0$, we seek solutions of the variable coefficient time-fractional diffusion equation away from the interface $\Gamma$ given by

$$
\frac{\partial^{\alpha} u(\boldsymbol{x}, t)}{\partial t^{\alpha}}-\nabla \cdot(\beta(\boldsymbol{x}, t) \nabla u(\boldsymbol{x}, t))=f(\boldsymbol{x}, t), \quad \boldsymbol{x} \in \Omega \backslash \Gamma, 0<t \leq T
$$

in which $\alpha$ is the order of the time-fractional derivative, $\boldsymbol{x}=(x, y)$ denotes the spatial variables and $\nabla$ is the gradient operator. The coefficient $\beta(\boldsymbol{x}, t)$ is assumed to be a $2 \times 2$ matrix that is uniformly elliptic on each disjoint subdomain, $\Omega^{-}$ and $\Omega^{+}$, and its components are continuously differentiable on each disjoint subdomain, but they may be discontinuous across the interface $\Gamma$. The right-hand side $f(\boldsymbol{x}, t)$ is assumed to lie in $L^{2}(\Omega)$.

Given functions $a$ and $b$ along the interface $\Gamma$, we prescribe the jump conditions

$$
\begin{aligned}
{[u]_{\Gamma}(\boldsymbol{x}, t) } & \equiv u^{+}(\boldsymbol{x}, t)-u^{-}(\boldsymbol{x}, t) \\
& =a(\boldsymbol{x}, t) \\
{[(\beta \nabla u) \cdot \boldsymbol{n}]_{\Gamma}(\boldsymbol{x}, t) } & \equiv \boldsymbol{n} \cdot\left(\beta^{+}(\boldsymbol{x}, t) \nabla u^{+}(\boldsymbol{x}, t)\right)-\boldsymbol{n} \cdot\left(\beta^{-}(\boldsymbol{x}, t) \nabla u^{-}(\boldsymbol{x}, t)\right) \\
& =b(\boldsymbol{x}, t)
\end{aligned}
$$

The " \pm " superscripts refer to limits taken from within the subdomains $\Omega^{ \pm}$. For ease of discussion in this section, and for accuracy test in the numerical examples, we assume that $a$ and $b$ are smooth on the closure of $\Omega$. 
The boundary condition is given as

$$
u(\boldsymbol{x}, t)=g(\boldsymbol{x}, t), \quad \boldsymbol{x} \in \partial \Omega, \quad 0<t \leq T
$$

for a given function $g$ on the boundary $\partial \Omega$.

Finally, we prescribe the initial condition as

$$
u(\boldsymbol{x}, 0)=u_{0}(\boldsymbol{x}), \quad \boldsymbol{x} \in \Omega .
$$

The main challenge of solving this time-fractional diffusion equation with discontinuous coefficients is how to deal with the fractional derivatives $\frac{\partial^{\alpha} u(\boldsymbol{x}, t)}{\partial t^{\alpha}}$. Here $\frac{\partial^{\alpha} u(\boldsymbol{x}, t)}{\partial t^{\alpha}}$ is the Caputo fractional derivatives of order $\alpha$.

Specifically, when $\alpha=0$, equation (1) turns out to be a model of elliptic equation with discontinuous coefficients on the interface,

$$
\left\{\begin{array}{l}
-\nabla \cdot(\beta(\boldsymbol{x}) \nabla u(\boldsymbol{x}))=f(\boldsymbol{x}), \quad \boldsymbol{x} \in \Omega \backslash \Gamma, \\
{[u]_{\Gamma}(\boldsymbol{x})=a(\boldsymbol{x}), \quad[(\beta \nabla u) \cdot \boldsymbol{n}]_{\Gamma}(\boldsymbol{x})=b(\boldsymbol{x}),} \\
u(\boldsymbol{x})=g(\boldsymbol{x}), \quad \boldsymbol{x} \in \partial \Omega .
\end{array}\right.
$$

please refer to (Hou et al., 2010) for more details.

When $\alpha=1$, equation (1) turns out to be a classical model of diffusion equation with discontinuous coefficients on the interface,

$$
\left\{\begin{array}{l}
\frac{\partial u(\boldsymbol{x}, t)}{\partial t}-\nabla \cdot(\beta(\boldsymbol{x}, t) \nabla u(\boldsymbol{x}, t))=f(\boldsymbol{x}, t), \quad \boldsymbol{x} \in \Omega \backslash \Gamma, 0<t \leq T \\
{[u]_{\Gamma}(\boldsymbol{x}, t)=a(\boldsymbol{x}, t), \quad[(\beta \nabla u) \cdot \boldsymbol{n}]_{\Gamma}(\boldsymbol{x}, t)=b(\boldsymbol{x}, t), \quad 0<t \leq T} \\
u(\boldsymbol{x}, t)=g(\boldsymbol{x}, t), \quad \boldsymbol{x} \in \partial \Omega, \quad 0<t \leq T \\
u(\boldsymbol{x}, 0)=u_{0}(\boldsymbol{x}), \quad \boldsymbol{x} \in \Omega
\end{array}\right.
$$

please see (Wang and Shi, 2015) for more details.

In this paper, the value of $\alpha$ under consideration is $0<\alpha<1$. In ( $\mathrm{Lin}$ and $\mathrm{Xu}$, 2007), the Caputo fractional derivative is defined as

$$
\frac{\partial^{\alpha} u(\boldsymbol{x}, t)}{\partial t^{\alpha}}=\frac{1}{\Gamma(1-\alpha)} \int_{0}^{t} \frac{\partial u(\boldsymbol{x}, s)}{\partial s} \frac{d s}{(t-s)^{\alpha}}, \quad 0<\alpha<1
$$

From this definition, it is obvious that the Caputo fractional derivatives uses the information of the standard derivatives at all previous time steps. Let $\Delta t=\frac{T}{K}$ be the time step, then $t_{k}=k \Delta t, k=0,1, \cdots, K$. For simplicity, we use $u^{k}$ to represent $u\left(t_{k}\right)$. Putting equations (1)-(5) together, equation (1) can be rewritten to the following full discrete equation

$$
\begin{aligned}
& d_{0} u^{k+1}-\Gamma(2-\alpha) \Delta t^{\alpha} \nabla \cdot\left(\beta^{k+1} \nabla u^{k+1}\right) \\
= & \Gamma(2-\alpha) \Delta t^{\alpha} f^{k+1}+d_{0} u^{k}-\sum_{j=0}^{k-1} d_{j+1} u^{k-j}+\sum_{j=1}^{k} d_{j} u^{k-j}, \\
& k=0,1, \cdots, K-1
\end{aligned}
$$

where $d_{j}=(j+1)^{1-\alpha}-(j)^{1-\alpha}, j=0,1, \cdots, k$ and $d_{0}=1$. According to (Lin and Xu, 2007), it is a (2- $\left.\alpha\right)$-order accuracy scheme.

Specifically, when $k=1$, equation (6) can be rewritten as

$$
d_{0} u^{2}-\Gamma(2-\alpha) \Delta t^{\alpha} \nabla \cdot\left(\beta^{2} \nabla u^{2}\right)=\Gamma(2-\alpha) \Delta t^{\alpha} f^{2}+\left(1-d_{1}\right) u^{1}+d_{1} u^{0},
$$

and when $k=0$, equation (6) can be rewritten as

$$
d_{0} u^{1}-\Gamma(2-\alpha) \Delta t^{\alpha} \nabla \cdot\left(\beta^{1} \nabla u^{1}\right)=\Gamma(2-\alpha) \Delta t^{\alpha} f^{1}+u^{0} .
$$


Multiplying both sides of equation (6) with the test function $\psi \in H_{0}^{1}(\Omega)$, integrating over each subdomain $\Omega^{+}$and $\Omega^{-}$, we deduce the weak formulation from Green's theorem of equation (6) with $u^{k}=g^{k}$ on boundary points as

$$
\begin{aligned}
& \int_{\Omega^{+}} d_{0} u^{k+1} \psi+\alpha_{0} \int_{\Omega^{+}} \beta^{k+1} \nabla u^{k+1} \cdot \nabla \psi+ \\
& \int_{\Omega^{-}} d_{0} u^{k+1} \psi+\alpha_{0} \int_{\Omega^{-}} \beta^{k+1} \nabla u^{k+1} \cdot \nabla \psi \\
= & \int_{\Omega^{+}}\left(d_{0} u^{k}-\sum_{j=0}^{k-1} d_{j+1} u^{k-j}+\sum_{j=1}^{k} d_{j} u^{k-j}\right) \psi+ \\
& \int_{\Omega^{-}}\left(d_{0} u^{k}-\sum_{j=0}^{k-1} d_{j+1} u^{k-j}+\sum_{j=1}^{k} d_{j} u^{k-j}\right) \psi+ \\
& \alpha_{0} \int_{\Omega} f^{k+1} \psi-\alpha_{0} \int_{\Gamma} b^{k+1} \psi, \\
& k=0,1, \cdots, K-1 .
\end{aligned}
$$

where $\alpha_{0}=\Gamma(2-\alpha) \Delta t^{\alpha}$.

\section{Numerical Method}

In this section, we are going to introduce our method for solving the two-dimensional time-fractional diffusion equation with interfaces using the Petrov-Galerkin finite element method in space and the finite difference method in time. By the definition of the fractional derivative and from equation (7), we can see that the solution at time $t_{k}$ depends on the solutions at all previous time steps. Since the problem under consideration is the time-fractional diffusion equation with interfaces, the coefficients $\beta(\boldsymbol{x}, t)$ and the jump conditions $a(\boldsymbol{x}, t)$ and $b(\boldsymbol{x}, t)$ in this paper all depend on time. In this section, we are going to construct and solve the local system.

For ease of discussion, we restrict ourselves to a rectangular domain $\Omega=\left(x_{\min }, x_{\max }\right) \times\left(y_{\min }, y_{\max }\right)$ in the plane. Given positive integers $I$ and $J$, set $\Delta x=\left(x_{\max }-x_{\min }\right) / I$ and $\Delta y=\left(y_{\max }-y_{\min }\right) / J$. Define a uniform Cartesian grid $\left(x_{i}, y_{j}\right)=$ $\left(x_{\min }+i \Delta x, y_{\min }+j \Delta y\right)$ for $i=0, \ldots, I$ and $j=0, \ldots, J$. Each $\left(x_{i}, y_{j}\right)$ is called a grid point. For the case $i=0, I$ or $j=0, J$, a grid point is called a boundary point, otherwise it is called an interior point. The grid size is defined as $h=\max \{\Delta x, \Delta y\}>0$.

Two sets of grid functions are needed and they are denoted by

$$
H_{ \pm}^{1, h}=\left\{\omega^{h}=\left(\omega_{i, j}\right): 0 \leq i \leq I, 0 \leq j \leq J\right\}
$$

and

$$
H_{0}^{1, h}=\left\{\omega^{h}=\left(\omega_{i, j}\right) \in H_{ \pm}^{1, h}: \omega_{i, j}=0 \text { if } i=0, I \text { or } j=0, J\right\} .
$$

Cut each rectangular region $\left[x_{i}, x_{i+1}\right] \times\left[y_{j}, y_{j+1}\right]$ into two pieces of right triangular regions: one is bounded by $x=x_{i}, y=y_{j}$ and $y=\frac{y_{j+1}-y_{j}}{x_{i}-x_{i+1}}\left(x-x_{i+1}\right)+y_{j}$, the other is bounded by $x=x_{i+1}, y=y_{j+1}$ and $y=\frac{y_{j+1}-y_{j}}{x_{i}-x_{i+1}}\left(x-x_{i+1}\right)+y_{j}$. Collecting all these triangular regions, we obtain a uniform triangulation $T^{h}: \bigcup_{K \in T^{h}} K$, see Fig.1. 


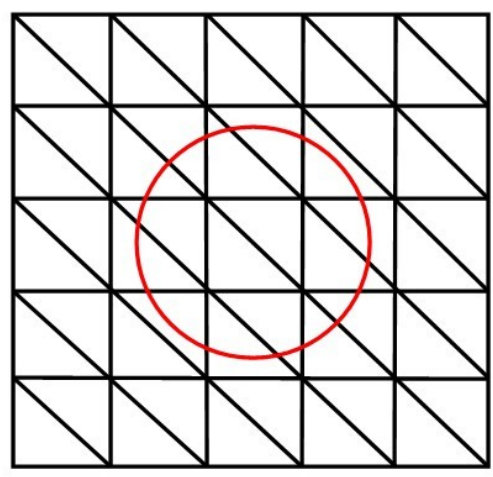

Figure 1. A uniform triangulation

If $\phi\left(x_{i}, y_{j}\right) \leq 0$, we count the grid point $\left(x_{i}, y_{j}\right)$ as in $\overline{\Omega^{-}}$; otherwise we count it as in $\Omega^{+}$. A cell $K$ is called a regular cell if all its vertices belong to the same subdomain, either $\Omega^{+}$or $\Omega^{-}$, otherwise it is called an interface cell. There are four kinds of interface cells, see Fig. 2. In an interface cell, we write $K=K^{+} \cup K^{-} . K^{+}$and $K^{-}$are separated by a straight line segment, denoted by $\Gamma_{K}^{h}$. The two end points of the line segment $\Gamma_{K}^{h}$ are located on interface $\Gamma$ and their locations can be calculated from the linear interpolations of the discrete level-set functions $\phi^{h}=\phi\left(x_{i}, y_{j}\right)$.

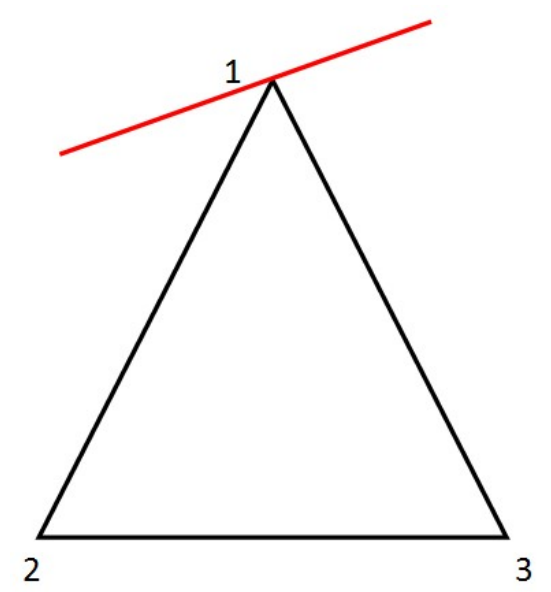

(a) The interface comes across one vertex of the triangle.

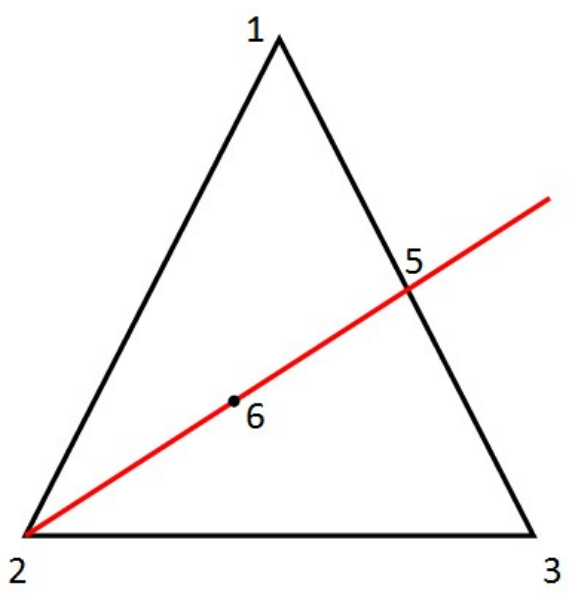

(c) The interface comes across one vertex and one side of the triangle.

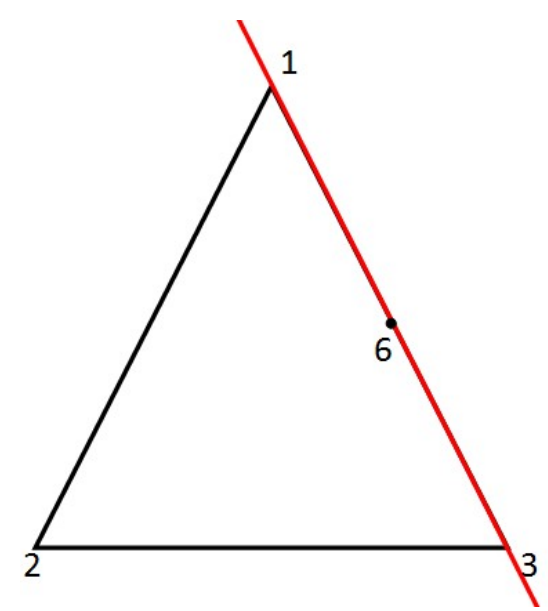

(b) The interface covers one side of the triangle.

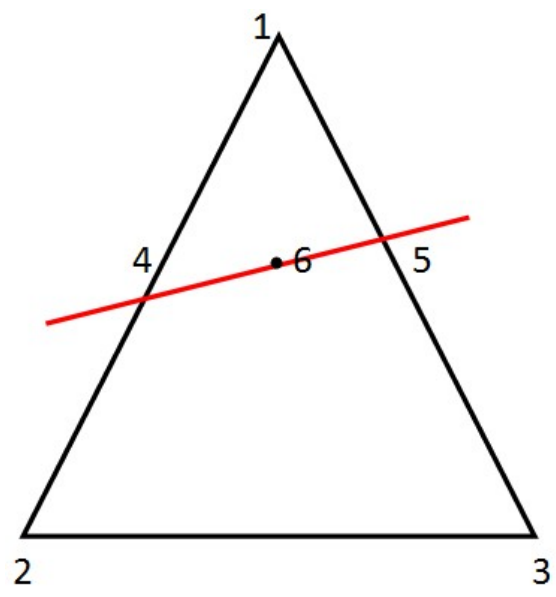

(d) The interface cuts two sides of the triangle.

Figure 2. Interface cells 
In the following discussion, two extension operators are needed. The first one is $T^{h}: H_{ \pm}^{1, h} \rightarrow H_{0}^{1}(\Omega)$. For any $\psi^{h} \in H_{0}^{1, h}$, $T^{h}\left(\psi^{h}\right)$ is a standard continuous piecewise linear function, which is a linear function in every triangular cell and $T^{h}\left(\psi^{h}\right)$ matches $\psi^{h}$ on grid points. Clearly such a function set, denoted by $H_{0}^{1, h}$, is a finite dimensional subspace of $H_{0}^{1}(\Omega)$. The second extension operator $U^{h}$ is constructed as follows: for any $u^{h, k} \in H_{ \pm}^{1, h}$ with $u^{h, k}=g^{h, k}$ at boundary points, $U^{h}\left(u^{h, k}\right)$ is a piecewise linear function and matches $u^{h, k}$ on grid points. It is a linear function in each regular cell, just like the first extension operator $U^{h}\left(u^{h, k}\right)=T^{h}\left(u^{h, k}\right)$ in a regular cell. In each interface cell, it consists of two pieces of linear functions, one is on $K^{+}$and the other is on $K^{-}$. The location of its discontinuity in the interface cell is the straight line segment $\Gamma_{K}^{h}$. Note that two end points of the line segment are located on interface $\Gamma$, hence the interface condition $[u]=a$ could be and is enforced exactly at these two end points. In each interface cell, the interface condition $[\beta \nabla u \cdot \boldsymbol{n}]=b$ is enforced with value $b$ at the middle point of $\Gamma_{K}^{h}$.

We shall construct an approximate solution to the interface problem taking into account the jump conditions. Note that the Neumann jump condition along the interface $\Gamma$ is already absorbed into the weak formulation, hence, we only need to take care of the Dirichlet jump condition along the interface $\Gamma$. We shall seek an approximate solution which is piecewise linear on both subdomains $\Omega^{-}$and $\Omega^{+}$, but discontinuous along the interface $\Gamma$. Clearly, in cases (a)-(c) of Fig. 2, when a vertex $(x, y)$ of the interface cell $K$ is on the interface, we need to get two solutions $u^{k}(x, y)=\left\{\begin{array}{l}u^{+, k}(x, y) \\ u^{-, k}(x, y)\end{array}\right.$ defined at the same point $(x, y)$ and at the same time $t_{k}$. We will compute one solution $u^{+, k}(x, y)$, and the other solution $u^{-, k}(x, y)$ can be derive from the Dirichlet jump condition, such that $u^{-, k}(x, y)=u^{+, k}(x, y)-a^{k}(x, y)$. To this end, we define $u^{k}(x, y)$ as follows:

$$
u^{k}(x, y)=\left\{\begin{array}{l}
u^{+, k}(x, y), \text { if } \phi(x, y) \geq 0, \\
u^{-, k}(x, y), \text { if } \phi(x, y)<0 .
\end{array}\right.
$$

Theorem 3.1. For all $u^{h, k} \in H_{ \pm}^{1, h}, U^{h}\left(u^{h, k}\right)$ can be constructed uniquely at $t_{k}$, provided $T^{h}, \phi, a^{k}$ and $b^{k}$ are given.

Proof. Based on the classification of cells, we take the following five different cases into consideration:

Case 0. For the regular cell $K, K \subset \Omega^{+}$or $K \subset \Omega^{-}$, depending on which subdomain all the vertices of $K$ belong to. For this kind of cells, no extra treatment is needed to construct $U^{h}\left(u^{h, k}\right)$. Clearly, $U^{h}\left(u^{h, k}\right)$ is a standard linear finite element function in a regular cell,

$$
U^{h}\left(u^{h, k}\right)=c_{1}^{ \pm, k} x+c_{2}^{ \pm, k} y+c_{3}^{ \pm, k},
$$

where the coefficients $c_{1}^{ \pm, k}, c_{2}^{ \pm, k}$ and $c_{3}^{ \pm, k}$ can be calculated by the following system,

$$
\left[\begin{array}{lll}
x_{1} & y_{1} & 1 \\
x_{2} & y_{2} & 1 \\
x_{3} & y_{3} & 1
\end{array}\right]\left[\begin{array}{c}
c_{1}^{ \pm, k} \\
c_{2}^{ \pm, k} \\
c_{3}^{ \pm, k}
\end{array}\right]=\left[\begin{array}{l}
u_{1}^{k} \\
u_{2}^{k} \\
u_{3}^{k}
\end{array}\right] .
$$

The superscript + or - here depends on the subdomain $\Omega^{+}$or $\Omega^{-}$the cell $K$ belongs to.

Case 1. For the case when the interface comes across one vertex of the cell $K, K \subset \Omega^{+}$or $K \subset \Omega^{-}, U^{h}\left(u^{h, k}\right)$ can be construct as follows,

$$
U^{h}\left(u^{h, k}\right)=c_{1}^{ \pm, k} x+c_{2}^{ \pm, k} y+c_{3}^{ \pm, k} .
$$

Before calculating the coefficients $c_{1}^{ \pm, k}, c_{2}^{ \pm, k}$ and $c_{3}^{ \pm, k}$, we need to check whether an vertex is on the interface or not. If it is on the interface, then it belongs to $\Omega^{+}$, otherwise it depends on which domain it belongs to. From Fig. 2(a) we can see that point $p_{1}$ is on the interface. If $p_{2}$ and $p_{3}$ are in $\Omega^{+}$, then $K \subset \Omega^{+}$. The coefficients $c_{1}^{+, k}, c_{2}^{+, k}$ and $c_{3}^{+, k}$ can be calculated by the following system,

$$
\left[\begin{array}{lll}
x_{1} & y_{1} & 1 \\
x_{2} & y_{2} & 1 \\
x_{3} & y_{3} & 1
\end{array}\right]\left[\begin{array}{c}
c_{1}^{+, k} \\
c_{2}^{+, k} \\
c_{3}^{+, k}
\end{array}\right]=\left[\begin{array}{l}
u_{1}^{k} \\
u_{2}^{k} \\
u_{3}^{k}
\end{array}\right] .
$$

Otherwise $K \subset \Omega^{-}$, we need to take the Dirichlet jump condition into consideration. Then the coefficients $c_{1}^{-, k}, c_{2}^{-, k}$ and $c_{3}^{-, k}$ will be calculated by the following system,

$$
\left[\begin{array}{lll}
x_{1} & y_{1} & 1 \\
x_{2} & y_{2} & 1 \\
x_{3} & y_{3} & 1
\end{array}\right]\left[\begin{array}{c}
c_{1}^{-, k} \\
c_{2}^{-, k} \\
c_{3}^{-, k}
\end{array}\right]=\left[\begin{array}{c}
u_{1}^{k}-a_{1}^{k} \\
u_{2}^{k} \\
u_{3}^{k}
\end{array}\right]
$$


Case 2. The interface covers one side of the cell $K, K \subset \Omega^{+}$or $K \subset \Omega^{-}$. For this kind of cells, $U^{h}\left(u^{h, k}\right)$ can be construct as follows,

$$
U^{h}\left(u^{h, k}\right)=c_{1}^{ \pm, k} x+c_{2}^{ \pm, k} y+c_{3}^{ \pm, k} .
$$

From Fig. 2(b) we can see that points $p_{1}$ and $p_{3}$ are both on the interface. If $p_{2}$ belongs to $\Omega^{+}$, then $K \subset \Omega^{+}$. The coefficients $c_{1}^{+, k}, c_{2}^{+, k}$ and $c_{3}^{+, k}$ can be calculated by the following system,

$$
\left[\begin{array}{lll}
x_{1} & y_{1} & 1 \\
x_{2} & y_{2} & 1 \\
x_{3} & y_{3} & 1
\end{array}\right]\left[\begin{array}{c}
c_{1}^{+, k} \\
c_{2}^{+, k} \\
c_{3}^{+, k}
\end{array}\right]=\left[\begin{array}{c}
u_{1}^{k} \\
u_{2}^{k} \\
u_{3}^{k}
\end{array}\right] .
$$

Otherwise $K \subset \Omega^{-}$, we need to take the Dirichlet jump condition into consideration. Then the coefficients $c_{1}^{-, k}, c_{2}^{-, k}$ and $c_{3}^{-, k}$ will be calculated by the following system,

$$
\left[\begin{array}{lll}
x_{1} & y_{1} & 1 \\
x_{2} & y_{2} & 1 \\
x_{3} & y_{3} & 1
\end{array}\right]\left[\begin{array}{c}
c_{1}^{-, k} \\
c_{2}^{-, k} \\
c_{3}^{-, k}
\end{array}\right]=\left[\begin{array}{c}
u_{1}^{k}-a_{1}^{k} \\
u_{2}^{k} \\
u_{3}^{k}-a_{3}^{k}
\end{array}\right] .
$$

In the last two cases, the interface cell $K$ is separated into two different pieces $K^{+}$and $K^{-}$, such that $K=K^{+} \cup K^{-}$. In these special cases, we need to take the Dirichlet jump condition and the Neumann jump condition into consideration. Special piecewise linear polynomials satisfying interface jump conditions are employed on this cell. The key idea of our method for this kind of interface cells is to construct a piecewise function by two linear polynomials defined on $\mathrm{K}^{+}$and $K^{-}$. Let $p_{6}$ be the middle point of the interface segment $\Gamma_{K}^{h}$ on this cell. In Fig. 2(c), $p_{6}$ is the middle point of $p_{2}$ and $p_{5}$, the Dirichlet jump condition is enforced at points $p_{5}$ and $p_{6}$, and the Neumann jump condition is enforced at point $p_{6}$. In Fig. 2(d), $p_{6}$ be the middle point of $p_{4}$ and $p_{5}$, the Dirichlet jump condition is enforced at points $p_{4}$ and $p_{5}$, and the Neumann jump condition is enforced at point $p_{6}$. Before continue our discussion, some of the following notations are needed,

$$
\begin{aligned}
& d_{1,6}^{+, k}=n_{x, 6} \beta_{11,6}^{+, k}+n_{y, 6} \beta_{21,6}^{+, k}, \\
& d_{2,6}^{+, k}=n_{x, 6} \beta_{12,6}^{+, k}+n_{y, 6} \beta_{22,6}^{+, k}, \\
& d_{1,6}^{-, k}=n_{x, 6} \beta_{11,6}^{-, k}+n_{y, 6} \beta_{21,6}^{-, k}, \\
& d_{2,6}^{-, k}=n_{x, 6} \beta_{12,6}^{-, k}+n_{y, 6} \beta_{22,6}^{-, k} .
\end{aligned}
$$

Case 3. The interface comes across one vertex and cuts one side of the cell $K . U^{h}\left(u^{h, k}\right)$ can be constructed as follows,

$$
U^{h}\left(u^{h, k}\right)=\left\{\begin{array}{l}
c_{1}^{+, k} x+c_{2}^{+, k} y+c_{3}^{+, k}, \text { if }(x, y) \in K^{+}, \\
c_{1}^{-, k} x+c_{2}^{-, k} y+c_{3}^{-, k}, \text { if }(x, y) \in K^{-} .
\end{array}\right.
$$

From Fig. 2(c) we can see that point $p_{2}$ is on the interface. If $p_{1}$ belongs to $\Omega^{+}$, then $K^{+}=K_{125} \subset \Omega^{+}$and $K^{-}=K_{235} \subset \Omega^{-}$. The coefficients $c_{1}^{ \pm, k}, c_{2}^{ \pm, k}$ and $c_{3}^{ \pm, k}$ can be calculated by the following system,

$$
\left[\begin{array}{cccccc}
x_{1} & y_{1} & 1 & 0 & 0 & 0 \\
x_{2} & y_{2} & 1 & 0 & 0 & 0 \\
0 & 0 & 0 & x_{3} & y_{3} & 1 \\
x_{5} & y_{5} & 1 & -x_{5} & -y_{5} & -1 \\
x_{6} & y_{6} & 1 & -x_{6} & -y_{6} & -1 \\
d_{1,6}^{+, k} & d_{2,6}^{+, k} & 0 & -d_{1,6}^{-, k} & -d_{2,6}^{-, k} & 0
\end{array}\right]\left[\begin{array}{c}
c_{1}^{+, k} \\
c_{2}^{+, k} \\
c_{3}^{+, k} \\
c^{-,} \\
c_{2}^{-, k} \\
c_{3}^{-,, k}
\end{array}\right]=\left[\begin{array}{c}
u_{1}^{k} \\
u_{2}^{k} \\
u_{3}^{k} \\
a_{5}^{k} \\
a_{6}^{k} \\
b_{6}^{k}
\end{array}\right],
$$

Otherwise $K^{-}=K_{125} \subset \Omega^{-}$and $K^{+}=K_{235} \subset \Omega^{+}$. Then the coefficients can be calculated by the following system,

$$
\left[\begin{array}{cccccc}
0 & 0 & 0 & x_{1} & y_{1} & 1 \\
x_{2} & y_{2} & 1 & 0 & 0 & 0 \\
x_{3} & y_{3} & 1 & 0 & 0 & 0 \\
x_{5} & y_{5} & 1 & -x_{5} & -y_{5} & -1 \\
x_{6} & y_{6} & 1 & -x_{6} & -y_{6} & -1 \\
d_{1,6}^{+, k} & d_{2,6}^{+, k} & 0 & -d_{1,6}^{-, k} & -d_{2,6}^{-, k} & 0
\end{array}\right]\left[\begin{array}{c}
c_{1}^{+, k} \\
c_{2}^{+, k} \\
c_{3}^{+, k} \\
c_{1}^{-, k} \\
c_{2}^{-, k} \\
c_{3}^{-, k}
\end{array}\right]=\left[\begin{array}{c}
u_{1}^{k} \\
u_{2}^{k} \\
u_{3}^{k} \\
a_{5}^{k} \\
a_{6}^{k} \\
b_{6}^{k}
\end{array}\right] .
$$


Case 4. The interface cuts two sides of the cell $K$, see Fig. 2(d). $U^{h}\left(u^{h, k}\right)$ can be constructed as follows,

$$
U^{h}\left(u^{h, k}\right)=\left\{\begin{array}{l}
c_{1}^{+, k} x+c_{2}^{+, k} y+c_{3}^{+, k}, \text { if }(x, y) \in K^{+}, \\
c_{1}^{-, k} x+c_{2}^{-, k} y+c_{3}^{-, k}, \text { if }(x, y) \in K^{-} .
\end{array}\right.
$$

If $p_{1}$ belongs to $\Omega^{+}$, then $K^{+}=K_{145} \subset \Omega^{+}$and $K^{-}=K_{4235} \subset \Omega^{-}$. The coefficients $c_{1}^{ \pm, k}, c_{2}^{ \pm, k}$ and $c_{3}^{ \pm, k}$ can be calculated by the following system,

$$
\left[\begin{array}{cccccc}
x_{1} & y_{1} & 1 & 0 & 0 & 0 \\
0 & 0 & 0 & x_{2} & y_{2} & 1 \\
0 & 0 & 0 & x_{3} & y_{3} & 1 \\
x_{4} & y_{4} & 1 & -x_{4} & -y_{4} & -1 \\
x_{5} & y_{5} & 1 & -x_{5} & -y_{5} & -1 \\
d_{1,6}^{+, k} & d_{2,6}^{+, k} & 0 & -d_{1,6}^{-, k} & -d_{2,6}^{-, k} & 0
\end{array}\right]\left[\begin{array}{c}
c_{1}^{+, k} \\
c_{2}^{+, k} \\
c_{3}^{+, k} \\
c^{-,} \\
c_{2}^{-, k} \\
c_{3}^{-,, k}
\end{array}\right]=\left[\begin{array}{c}
u_{1}^{k} \\
u_{2}^{k} \\
u_{3}^{k} \\
a_{4}^{k} \\
a_{5}^{k} \\
b_{6}^{k}
\end{array}\right] .
$$

Otherwise $K^{-}=K_{145} \subset \Omega^{-}$and $K^{+}=K_{4235} \subset \Omega^{+}$. Then the coefficients can be calculated by the following system,

$$
\left[\begin{array}{cccccc}
0 & 0 & 0 & x_{1} & y_{1} & 1 \\
x_{2} & y_{2} & 1 & 0 & 0 & 0 \\
x_{3} & y_{3} & 1 & 0 & 0 & 0 \\
x_{4} & y_{4} & 1 & -x_{4} & -y_{4} & -1 \\
x_{5} & y_{5} & 1 & -x_{5} & -y_{5} & -1 \\
d_{1,6}^{+, k} & d_{2,6}^{+, k} & 0 & -d_{1,6}^{-, k} & -d_{2,6}^{-, k} & 0
\end{array}\right]\left[\begin{array}{c}
c_{1}^{+, k} \\
c_{2}^{+, k} \\
c_{3}^{+, k} \\
c_{1}^{-, k} \\
c_{2}^{-, k} \\
c_{3}^{-, k}
\end{array}\right]=\left[\begin{array}{c}
u_{1}^{k} \\
u_{2}^{k} \\
u_{3}^{k} \\
a_{4}^{k} \\
a_{5}^{k} \\
b_{6}^{k}
\end{array}\right] .
$$

From Theorem 3.1 we can see that the gradient of $u$ on a cell $K$ can be represented by a linear combination of $u_{1}^{k}, u_{2}^{k}, u_{3}^{k}$, $a_{4}^{k}, a_{5}^{k}$ and $b_{6}^{k}$ :

$$
\begin{aligned}
& \nabla u^{ \pm, k} \\
= & {\left[\begin{array}{l}
u_{x}^{ \pm, k} \\
u_{y}^{ \pm, k}
\end{array}\right]=\left[\begin{array}{c}
c_{1}^{ \pm, k} \\
c_{2}^{ \pm, k}
\end{array}\right] } \\
= & {\left[\begin{array}{c}
c_{x, 1}^{ \pm, k} u_{1}^{k}+c_{x, 2}^{ \pm, k} u_{2}^{k}+c_{x, 3}^{ \pm, k} u_{3}^{k}+c_{x, 4}^{ \pm, k} a_{4}^{k}+c_{x, 5}^{ \pm, k} a_{5}^{k}+c_{x, 5}^{ \pm, k} a_{6}^{k}+c_{x, 6}^{ \pm, k} b_{6}^{k} \\
c_{y, 1}^{ \pm, k} u_{1}^{k}+c_{y, 2}^{ \pm, k} u_{2}^{k}+c_{y, 3}^{ \pm, k} u_{3}^{k}+c_{y, 4}^{ \pm, k} a_{4}^{k}+c_{y, 5}^{ \pm, k} a_{5}^{k}+c_{y, 5}^{ \pm, k} a_{6}^{k}+c_{y, 6}^{ \pm, k} b_{6}^{k}
\end{array}\right] . }
\end{aligned}
$$

Clearly, in Cases 0, 1 and 2, the coefficients of $a_{4}^{k}, a_{5}^{k}, a_{6}^{k}$ and $b_{6}^{k}$ are 0 ; in Case 3, the coefficient of $a_{4}^{k}$ is 0 ; in Case 4, and the coefficient of $a_{6}^{k}$ is 0 .

Based on the above discussion, we derive the following Theorem:

Theorem 3.2. All coefficients $c^{k}$ in equation (8) are finite and independent of $u^{k}, a^{k}$ and $b^{k}$.

For simplicity, we define some notations. Let

$$
\begin{aligned}
I_{u}^{k}(K) & =\int_{K} U^{h, k}\left(u^{h, k}\right) T^{h}\left(\psi^{h}\right), \\
I_{\nabla u}^{k}(K) & =\int_{K} \beta \nabla U^{h, k}\left(u^{h, k}\right) \cdot \nabla T^{h}\left(\psi^{h}\right), \\
I_{d_{j+1}}^{k}(K) & =\int_{K}\left(\sum_{j=0}^{k-1} d_{j+1} U^{h, k-j}\left(u^{h, k-j}\right) T^{h}\left(\psi^{h}\right)\right), \\
I_{d_{j}}^{k}(K) & =\int_{K}\left(\sum_{j=1}^{k} d_{j} U^{h, k-j}\left(u^{h, k-j}\right) T^{h}\left(\psi^{h}\right)\right), \\
I_{f}^{k}(K) & =\int_{K} f^{k} T^{h}\left(\psi^{h}\right), \\
I_{b}^{k}\left(\Gamma_{K}^{h}\right) & =\int_{\Gamma_{K}^{h}} b^{k} T^{h}\left(\psi^{h}\right) .
\end{aligned}
$$


After the construction of $U^{h}\left(u^{h, k}\right)$ and with the notations defined in equation (9), we further derive the integral equation defined on cell $K$. Again, we deduce the integral equation for each case mentioned in Theorem 3.1.

In Cases 0 and 1, the integral equation can be derived directly without any special treatment,

$$
\begin{aligned}
& d_{0} I_{u}^{k+1}\left(K^{ \pm}\right)+\alpha_{0} I_{\nabla u}^{k+1}\left(K^{ \pm}\right) \\
= & d_{0} I_{u}^{k}\left(K^{ \pm}\right)-I_{d_{j+1}}^{k}\left(K^{ \pm}\right)+I_{d_{j}}^{k}\left(K^{ \pm}\right)+\alpha_{0} I_{f}^{k+1}\left(K^{ \pm}\right),
\end{aligned}
$$

The sign of the superscript depends on which subdomain the cell $K$ belongs to, $\Omega^{+}$or $\Omega^{-}$.

The difference between Cases 1 and 2 is that when calculating the integration, Case 1 does not need to calculate the line integration, but Case 2 does. This means in Case 2 the Neumann jump condition needs to be taken into consideration. When $K \subset \Omega^{+}$, we have

$$
\begin{aligned}
& d_{0} I_{u}^{k+1}\left(K^{+}\right)+\alpha_{0} I_{\nabla u}^{k+1}\left(K^{+}\right) \\
= & d_{0} I_{u}^{k}\left(K^{+}\right)-I_{d_{j+1}}^{k}\left(K^{+}\right)+I_{d_{j}}^{k}\left(K^{+}\right)+\alpha_{0} I_{f}^{k+1}\left(K^{+}\right),
\end{aligned}
$$

when $K \subset \Omega^{-}$, we have

$$
\begin{aligned}
& d_{0} I_{u}^{k+1}\left(K^{-}\right)+\alpha_{0} I_{\nabla u}^{k+1}\left(K^{-}\right) \\
= & d_{0} I_{u}^{k}\left(K^{-}\right)-I_{d_{j+1}}^{k}\left(K^{-}\right)+I_{d_{j}}^{k}\left(K^{-}\right)+\alpha_{0} I_{f}^{k+1}\left(K^{-}\right)-\alpha_{0} I_{b}^{k+1}\left(\Gamma_{K}^{h}\right),
\end{aligned}
$$

In Cases 3 and 4, the cell $K$ is separated into two pieces, $K=K^{+} \cup K^{-}$, hence the integration on this cell is conducted in two separated pieces $K^{+}$and $K^{-}$,

$$
\begin{aligned}
& d_{0} I_{u}^{k+1}\left(K^{+}\right)+d_{0} I_{u}^{k+1}\left(K^{-}\right)+\alpha_{0} I_{\nabla u}^{k+1}\left(K^{+}\right)+\alpha_{0} I_{\nabla u}^{k+1}\left(K^{-}\right) \\
= & d_{0} I_{u}^{k}\left(K^{+}\right)-I_{d_{j+1}}^{k}\left(K^{+}\right)+I_{d_{j}}^{k}\left(K^{+}\right)+\alpha_{0} I_{f}^{k+1}\left(K^{+}\right)-\alpha_{0} I_{b}^{k+1}\left(\Gamma_{K}^{h}\right)+ \\
& d_{0} I_{u}^{k}\left(K^{-}\right)-I_{d_{j+1}}^{k}\left(K^{-}\right)+I_{d_{j}}^{k}\left(K^{-}\right)+\alpha_{0} I_{f}^{k+1}\left(K^{-}\right)
\end{aligned}
$$

Based on the above discussion, collecting the integration on every different cells (see equations (10)-(13)) together, we propose the following method:

Method 3.1. Find a discrete function $u^{h, k} \in H_{ \pm}^{1, h}$ with $u^{h, k}=g^{h, k}$ on boundary points so that for all $\psi^{h} \in H_{0}^{1, h}$, we have

$$
\begin{aligned}
& \sum_{K \in T^{h}}\left(d_{0} I_{u}^{k+1}\left(K^{+}\right)+d_{0} I_{u}^{k+1}\left(K^{-}\right)+\alpha_{0} I_{\nabla u}^{k+1}\left(K^{+}\right)+\alpha_{0} I_{\nabla u}^{k+1}\left(K^{-}\right)\right) \\
= & \sum_{K \in T^{h}}\left(d_{0} I_{u}^{k}\left(K^{+}\right)-I_{d_{j+1}}^{k}\left(K^{+}\right)+I_{d_{j}}^{k}\left(K^{+}\right)+\alpha_{0} I_{f}^{k+1}\left(K^{+}\right)+\right. \\
& \left.d_{0} I_{u}^{k}\left(K^{-}\right)-I_{d_{j+1}}^{k}\left(K^{-}\right)+I_{d_{j}}^{k}\left(K^{-}\right)+\alpha_{0} I_{f}^{k+1}\left(K^{-}\right)-\alpha_{0} I_{b}^{k+1}\left(\Gamma_{K}^{h}\right)\right)
\end{aligned}
$$

Remark 3.1. In our implementation, the integrals are computed with Gaussian quadrature rules. Since the interface can separate a cell into many different polygon, when calculating the integral, we further cut it into a few triangles. For each triangular cell, the midpoint of each edge is denoted by $p_{i j}$. In numerical computation, the average of three $f\left(p_{i j}\right)$ in each cell is utilized.

\section{Numerical Experiments}

In all numerical experiments below, the level-set function $\phi(x, y)$, the coefficients $\beta^{ \pm}(x, y, t)$ and the solutions

$$
u=\left\{\begin{array}{l}
u^{+}(x, y, t), \text { on } \Omega^{+}, \\
u^{-}(x, y, t), \text { on } \Omega^{-}
\end{array}\right.
$$

are given. Hence

$$
\begin{aligned}
& u_{0}(x, y)=\left\{\begin{array}{l}
u^{+}(x, y, 0), \text { in } \Omega^{+}, \\
u^{-}(x, y, 0), \text { in } \Omega^{-},
\end{array}\right. \\
& g(x, y, t)=\left\{\begin{array}{l}
u^{+}(x, y, t), \text { in } \partial \Omega^{+}, \\
u^{-}(x, y, t), \text { in } \partial \Omega^{-},
\end{array}\right. \\
& f(x, y, t)=\left\{\begin{array}{l}
\frac{\partial^{\alpha} u^{+}(x, y, t)}{\partial \partial^{\alpha}}-\nabla \cdot\left(\beta^{+}(x, y, t) \nabla u^{+}(x, y, t)\right), \text { in } \Omega^{+}, \\
\frac{\partial^{\alpha} u^{-}(x, y, t)}{\partial t^{\alpha}}-\nabla \cdot\left(\beta^{-}(x, y, t) \nabla u^{-}(x, y, t)\right), \text { in } \Omega^{-},
\end{array}\right. \\
& a(x, y, t)=u^{+}(x, y, t)-u^{-}(x, y, t) \text {, on } \Gamma \text {, } \\
& b(x, y, t)=\left(\beta^{+}(x, y, t) \nabla u^{+}(x, y, t)\right) \cdot \boldsymbol{n}-\left(\beta^{-}(x, y, t) \nabla u^{-}(x, y, t)\right) \cdot \boldsymbol{n}, \text { on } \Gamma
\end{aligned}
$$


All the examples are defined on the domain $[0,1] \times[-1,1] \times[-1,1]$. All errors in solutions are measured in the $L^{\infty}$ norm and the $L^{2}$ norm in the whole domain $\Omega$.

Example 1. This example is a "happy face" interface. The level-set function $\phi$, the coefficients $\beta^{ \pm}$and the solution $u^{ \pm}$are given as follows:

$$
\begin{aligned}
\phi(x, y) & =\max \left(\min \left(\phi_{1}, \phi_{2}, \phi_{3}\right), \phi_{4}, \phi_{5}, \phi_{6}, \min \left(\phi_{7}, \phi_{8}\right)\right), \\
\phi_{1}(x, y) & =x^{2}+y^{2}-0.75^{2}-0.15^{2}, \\
\phi_{2}(x, y) & =(x-0.75)^{2}+y^{2}-0.15^{2}, \\
\phi_{3}(x, y) & =(x+0.75)^{2}+y^{2}-0.15^{2}, \\
\phi_{4}(x, y) & =-\frac{0.1}{0.12}(x-0.2)^{2}-\frac{0.12}{0.1}(y-0.22)^{2}+0.12 \cdot 0.1, \\
\phi_{5}(x, y) & =-\frac{0.1}{0.12}(x+0.2)^{2}-\frac{0.12}{0.1}(y-0.22)^{2}+0.12 \cdot 0.1, \\
\phi_{6}(x, y) & =-x^{2}-(y+0.08)^{2}+0.12^{2}, \\
\phi_{7}(x, y) & =-x^{2}-(y+0.625)^{2}+0.425^{2}, \\
\phi_{8}(x, y) & =-x^{2}-(y+0.25)^{2}+0.2^{2}, \\
\beta^{+}(x, y, t) & =\left(\begin{array}{cc}
(x+2) t & 0 \\
0 & 2+x y+t
\end{array}\right), \\
\beta^{-}(x, y, t) & =\left(\begin{array}{cc}
(y+2) t & 0 \\
0 & 0.5(x+y)+2+t
\end{array}\right), \\
u^{+}(x, y, t) & =t^{2} x y\left(1+x^{2}\right)(1-y), \\
u^{-}(x, y, t) & =t^{1.1} \sin (\pi x) \exp (2 y)
\end{aligned}
$$

In this example, the Caputo fractional derivatives $\frac{\partial^{\alpha} u^{ \pm}(x, y, t)}{\partial t^{t^{\gamma}}}$ in the corresponding forcing term $f(x, y, t)$ are

$$
\begin{aligned}
& \frac{\partial^{\alpha} u^{+}(x, y, t)}{\partial t^{\alpha}}=\frac{2 t^{2-\alpha}}{\Gamma(3-\alpha)} x y\left(1+x^{2}\right)(1-y) \\
& \frac{\partial^{\alpha} u^{-}(x, y, t)}{\partial t^{\alpha}}=\Gamma(2+\alpha) t \sin (\pi x) \exp (2 y)
\end{aligned}
$$

Figure 3 shows the numerical result of $\alpha=0.1, t=1$ using a $10 \times 40 \times 40$ grid. Table 1 shows the numerical result of $\alpha=0.1, t=1$.

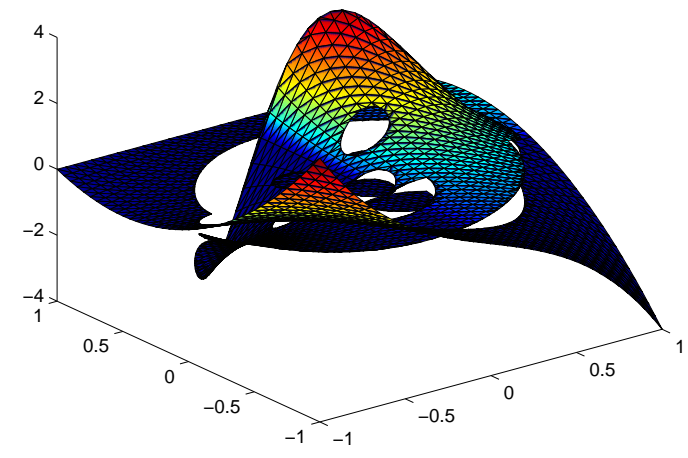

(a) Numerical result of $\alpha=0.1, t=1$ using a $10 \times 40 \times 40$ grid

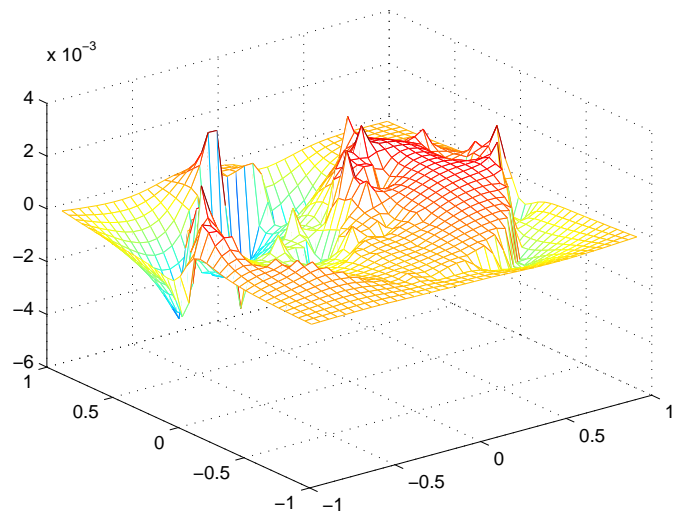

(b) Error of $\alpha=0.1, t=1$ using a $10 \times 40 \times 40$ grid

Figure 3. Numerical results for example 1 
Table 1. Numerical results for example 1 using four kinds of grids

\begin{tabular}{|c|c|c|c|c|}
\hline$\alpha$ & \multicolumn{4}{|c|}{0.1} \\
\hline$n_{t} \times n_{x} \times n_{y}$ & $\left\|u^{h}-u\right\|_{\infty}$ & Order & $\left\|u^{h}-u\right\|_{2}$ & Order \\
\hline $5 \times 20 \times 20$ & 0.020 & & 0.0094 & \\
$10 \times 40 \times 40$ & 0.0054 & 1.86 & 0.0020 & 2.21 \\
$20 \times 80 \times 80$ & 0.0014 & 1.92 & 0.00056 & 1.87 \\
$40 \times 160 \times 160$ & 0.00036 & 2.02 & 0.00013 & 2.14 \\
\hline
\end{tabular}

Example 2. This example is a "chess" interface. The level-set function $\phi$, the coefficients $\beta^{ \pm}$and the solution $u^{ \pm}$are given as follows:

$$
\begin{aligned}
\phi(x, y) & =-(\sin (5 \pi x)-y)(-\sin (5 \pi y)-x), \\
\beta^{+}(x, y, t) & =\left(\begin{array}{cc}
t x^{2}+3 & x^{2}+t / 5 \\
x^{2}+t / 5 & 2 t+x y+5
\end{array}\right), \\
\beta^{-}(x, y, t) & =\left(\begin{array}{cc}
t(y+2)+3 & y^{2}+t / 5 \\
y^{2}+t / 5 & x^{2} / 2+y^{2} / 2+t / 2+6
\end{array}\right), \\
u^{+}(x, y, t) & =t^{2} \sin (\pi x) \cos (\pi y), \\
u^{-}(x, y, t) & =t^{2.6} \exp (x+y+2)
\end{aligned}
$$

In this example, the Caputo fractional derivatives $\frac{\partial^{\alpha} u^{ \pm}(x, y, t)}{\partial t^{t^{a}}}$ in the corresponding forcing term $f(x, y, t)$ are

$$
\begin{aligned}
& \frac{\partial^{\alpha} u^{+}(x, y, t)}{\partial t^{\alpha}}=\frac{2 t^{2-\alpha}}{\Gamma(3-\alpha)} \sin (\pi x) \cos (\pi y) \\
& \frac{\partial^{\alpha} u^{-}(x, y, t)}{\partial t^{\alpha}}=\frac{\Gamma(3+\alpha)}{2} t^{2} \exp (x+y+2)
\end{aligned}
$$

Figure 4 shows the numerical result of $\alpha=0.6, t=1$ using a $10 \times 40 \times 40$ grid. Table 2 shows the numerical result of $\alpha=0.6, t=1$.

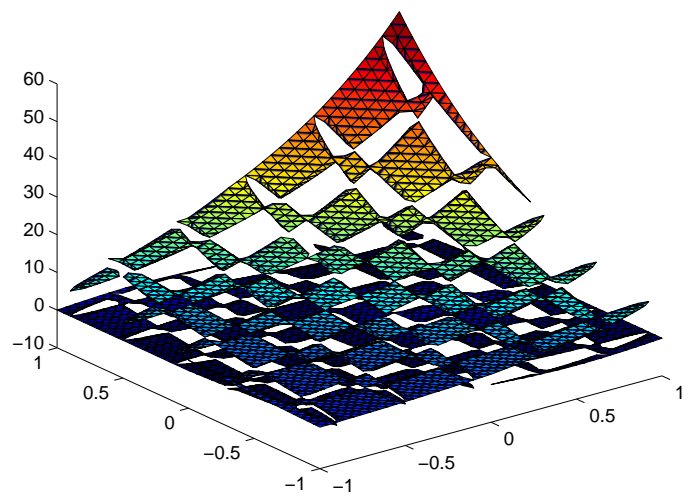

(a) Numerical Result of $\alpha=0.6, t=1$ using a $10 \times 40 \times 40$ grid

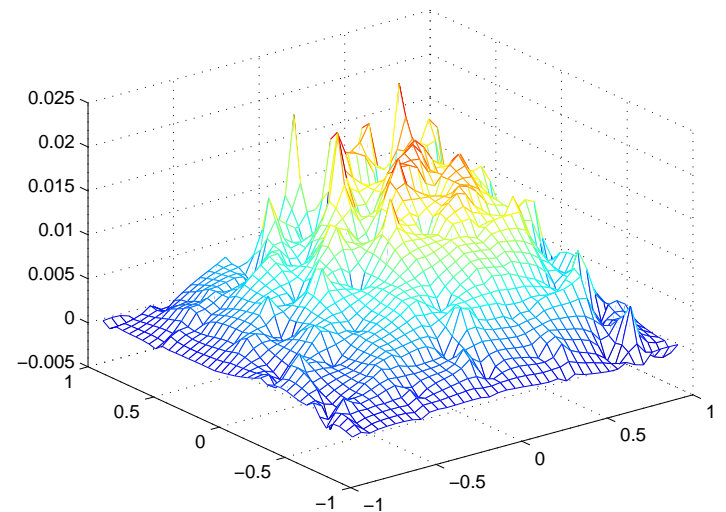

(b) Error of $\alpha=0.6, t=1$ using a $10 \times 40 \times 40$ grid

Figure 4. Numerical results for example 2

Example 3. This example is a "star" interface. The level-set function $\phi$, the coefficients $\beta^{ \pm}$and the solution $u^{ \pm}$are given as follows:

$$
\phi(r, \theta)= \begin{cases}\frac{R \sin \left(\frac{\theta_{t}}{2}\right)}{\sin \left(\frac{\theta_{t}}{2}+\theta-\theta_{r}-\frac{2 \pi(i-1)}{5}\right)}-r, & \theta_{r}+\frac{\pi(2 i-2)}{5} \leq \theta<\theta_{r}+\frac{\pi(2 i-1)}{5}, \\ \frac{R \sin \left(\frac{\theta_{t}}{2}\right)}{\sin \left(\frac{\theta_{t}}{2}-\theta+\theta_{r}-\frac{2 \pi(i-1)}{5}\right)}-r, & \theta_{r}+\frac{\pi(2 i-3)}{5} \leq \theta<\theta_{r}+\frac{\pi(2 i-2)}{5},\end{cases}
$$


Table 2. Numerical results for example 2 using four kinds of grids

\begin{tabular}{|c|c|c|c|c|}
\hline$\alpha$ & \multicolumn{4}{|c|}{0.6} \\
\hline$n_{t} \times n_{x} \times n_{y}$ & $\left\|u^{h}-u\right\|_{\infty}$ & Order & $\left\|u^{h}-u\right\|_{2}$ & Order \\
\hline $5 \times 20 \times 20$ & 0.062 & & 0.040 & \\
$10 \times 40 \times 40$ & 0.021 & 1.53 & 0.015 & 1.43 \\
$20 \times 80 \times 80$ & 0.0073 & 1.56 & 0.0053 & 1.48 \\
$40 \times 160 \times 160$ & 0.0026 & 1.46 & 0.0020 & 1.44 \\
\hline
\end{tabular}

with $\theta_{t}=\pi / 5, \theta_{r}=\pi / 7, R=6 / 7$ and $i=1,2,3,4,5$.

$$
\begin{aligned}
\beta^{+}(x, y, t) & =\left(\begin{array}{cc}
t x^{2}+3 & 0 \\
0 & 2 t+x y+5
\end{array}\right) \\
\beta^{-}(x, y, t) & =\left(\begin{array}{cc}
t(y+2)+3 & 0 \\
0 & x^{2} / 2+y^{2} / 2+t / 2+6
\end{array}\right), \\
u^{+}(x, y, t) & =t^{2.8}\left(x^{2}+y^{2}\right), \\
u^{-}(x, y, t) & =t^{1.8} \cos (x y)
\end{aligned}
$$

In this example, the Caputo fractional derivatives $\frac{\partial^{\alpha} u^{ \pm}(x, y, t)}{\partial t^{\alpha}}$ in the corresponding forcing term $f(x, y, t)$ are

$$
\begin{aligned}
\frac{\partial^{\alpha} u^{+}(x, y, t)}{\partial t^{\alpha}} & =\frac{\Gamma(3+\alpha)}{2} t^{2}\left(x^{2}+y^{2}\right) \\
\frac{\partial^{\alpha} u^{-}(x, y, t)}{\partial t^{\alpha}} & =\Gamma(2+\alpha) t \cos (x y)
\end{aligned}
$$

Figure 5 shows the numerical result of $\alpha=0.8, t=1$ using a $10 \times 40 \times 40$ grid. Table 3 shows the numerical result of $\alpha=0.8, t=1$.

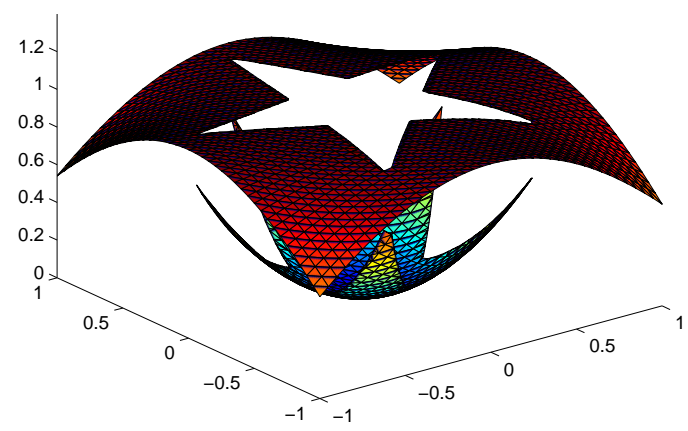

(a) Numerical result of $\alpha=0.8, t=1$ using a $10 \times 40 \times 40$ grid

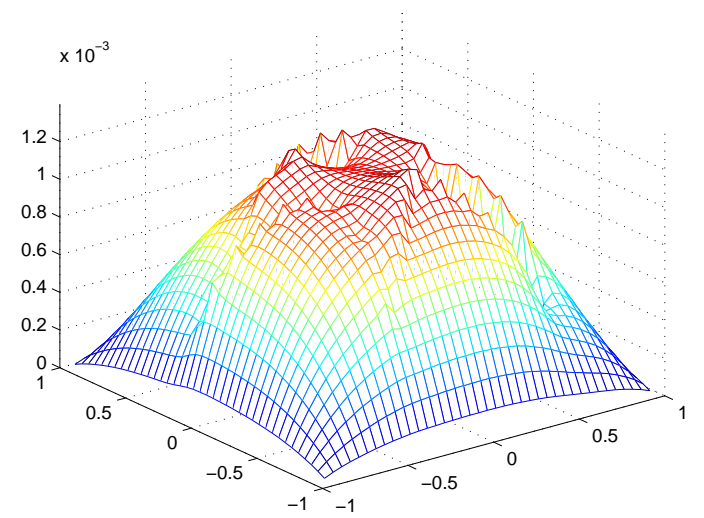

(b) Error of $\alpha=0.8, t=1$ using a $10 \times 40 \times 40$ grid

Figure 5. Numerical results for example 3

Table 3. Numerical results for example 3 using four kinds of grids

\begin{tabular}{|c|c|c|c|c|}
\hline$\alpha$ & \multicolumn{4}{|c|}{0.8} \\
\hline$n_{t} \times n_{x} \times n_{y}$ & $\left\|u^{h}-u\right\|_{\infty}$ & Order & $\left\|u^{h}-u\right\|_{2}$ & Order \\
\hline $5 \times 20 \times 20$ & 0.0030 & & 0.0031 & \\
$10 \times 40 \times 40$ & 0.0012 & 1.28 & 0.0014 & 1.12 \\
$20 \times 80 \times 80$ & 0.00054 & 1.20 & 0.00064 & 1.15 \\
$40 \times 160 \times 160$ & 0.00024 & 1.19 & 0.00028 & 1.17 \\
\hline
\end{tabular}




\section{Conclusion}

In this paper, we proposed a numerical method for solving the two-dimensional time-fractional diffusion equation with interface. Finite difference method and finite element method are employed to obtain the numerical solution in time and space. This method is capable of solving variable matrix coefficient problems with sharp-edged interfaces. We used several examples to show that our method has $(2-\alpha)$ order accuracy in both the $L^{2}$ and $L^{\infty}$ norm.

\section{References}

Baeumer, B., Benson, D. A., \& Meerschaert, M. M. (2005). Advection and dispersion in time and space. Physica A., 350, $245 \mathrm{C} 262$.

Burrage, K., Hale, N., \& Kay, D. (2012). An efficient implicit fem scheme for fractional-in-space reaction-diffusion equations. SIAM J. Sci. Comput., 34, 2145C2172.

Chadam, S. M., \& Yin, H. (1993). A diffusion equation with localized chemical reactions. Proc. of Edinburgh Math, 37, $101 \mathrm{C} 118$

Chen, Z., \& Zou, J. (1998). Finite element methods and their convergence for elliptic and parabolic interface problems. Numerische Mathematik., 79, 175C202.

Colella, P., \& Johansen, H. (1998). A cartesian grid embedded boundary method for poissons equation on irregular domains. J. Comput. Phys., 147, 60C85.

Dehghan, M., Abbaszadeh, M., \& Mohebbi, A. (2015). An implicit rbf meshless approach for solving the time fractional nonlinear sine-gordon and klein-gordon equations. Engineering Analysis with Boundary Elements., 50, $412 \mathrm{C} 434$.

Deng, W. (2008). Finite element method for the space and time fractional fokker-planck equation. SIAM J. Numer. Anal., 47, 204C226.

Duan, J. S. (2005). Time- and space-fractional partial differential equations. J. Math. Phys., 46(1), 13504C13511.

El-Wakil, S., Abulwafa, E., Zahran, M., \& Mahmoud, A. (2009). Time-fractional kdv equation, formulation and solution using variational methods. Physica Scripta., 65(1-2), 55 C63.

Ervin, V. J., Heuer, N., \& Roop, J. P. (2007). Numerical approximation of a time dependent, nonlinear, space-fractional diffusion equation. SIAM J. Numer. Anal., 45, 572C591.

Fan, W., Liu, F., Jiang, X., \& Turner, I. (2017). A novel unstructured mesh finite element method for solving the timespace fractional wave equation on a two-dimensional irregular convex domain. Fractional Calculus and Applied Analysis., 20(2), 352C383.

Fedkiw, R., Aslam, T., Merriman, B., \& Osher, S. (1999). A non-oscillatory eulerian approach to interfaces in multimaterial flows (the ghost fluid method). J. Comput. Phys., 152(2), 457C492.

Hou, S., \& Liu, X. D. (2005). A numerical method for solving variable coefficient elliptic equations with interfaces. $J$. Comput. Phys., 202, 411C445.

Hou, S., Wang, W., \& Wang, L. (2010). Numerical method for solving matrix coefficient elliptic equation with sharpedged interfaces. Comput. Phys., 229, 7162C7179.

Huang, F., \& Liu, F. (2005). The fundamental solution of the space-time fractional advection-dispersion equation. J. Appl. Math. Comput., 18(1-2), 339C350.

Ichise, M., Nagayanagi, Y., \& Kojima, T. (1971). An analog simulation of non-inteer order transfer functions for analysis of electrode processes. J. Electroanal. Chem., 33(2), 253C265.

Ji, X., \& Tang, H. (2012). High-order accurate runge-kutta (local) discontinuous galerkin methods for one-and twodimensional fractional diffusion equations. Numer. Math. Theory Methods Appl., 5, 333C358.

Jiang, R., \& Qi, H. (2010). Analytical solutions for anomalous transport of volatile pollutants in nonaqueous-phase liquid contaminated soil. Nonlinear Dyn., 62(4), 895C904.

Jiang, Y., \& Ma, J. (2011). High-order finite element methods for time-fractional partial differential equations. J. Comput. Appl. Math., 235(11), 3285C3290.

Justin, W. L. W., \& Liu, X. D. (2004). A boundary condition capturing multigrid approach to irregular boundary problems. SIAM J. Sci. Comput., 25(6), 1982C2003.

Kandilarov, J. D. (2005). A rothe-immersed interface method for a class of parabolic interface problems. Numer. Anal. 
Appl., 3401, 328C336.

Langlands, T. A. M., \& Henry, B. I. (2005). The accuracy and stability of an implicit solution method for the fractional diffusion equation. J. Comput. Phys., 205, 719C736.

LeVeque, R. J., \& Li, Z. (1994). The immersed interface method for elliptic equations with discontinuous coefficients and singular sources. SIAM J. Numer. Anal., 31, 1019C1044.

Li, X., \& Xu, C. (2009). A space-time spectral method for the time fractional diffusion equation. SIAM J. Numer. Anal., $47(3), 2108 \mathrm{C} 2131$.

Lin, Y., \& Xu, C. (2007). Finite difference/spectral approximations for the time-fractional diffusion equation. J. Comput. Phys., 225, 1533C1552.

Liu, F., Anh, V., \& Turner, I. (2002). Numerical solution of the fractional-order advection-dispersion equation. Proceedings of the International Conference on Boundary and Interior Layers, Perth, Australia, 159C164.

Liu, F., Anh, V., Turner, I., \& Zhuang, P. (2003). The fractional advection-dispersion equation. J. Appl. Math. Comput., 13(1), $233 \mathrm{C} 245$.

Liu, F., Anh, V. V., \& Turner, I. W. (2004). Numerical solution of the space fractional fokker-planck equation. J. Comput. Appl. Math., 166, 209C219.

Liu, F., Zhuang, P., Anh, V., Turner, T., \& Burrage, K. (2007). Stability and convergence of the difference methods for the space-time fractional advection-diffusion equation. Appl. Math. Comput., 191, 12C20.

Liu, Q., Gu, Y., Zhuang, P., Liu, F., \& Nie, Y. (2011). An implicit rbf meshless approach for time fractional diffusion equations. Comput. Mech., 48(1), $1 \mathrm{C} 12$.

Liu, X. D., Fedkiw, R. P., \& Myungjoo, K. (2000). A boundary condition capturing method for poissons equation on irregular domains. J. Comput. Phys., 160(1), 151C178.

Macklin, P. \& Lowengrub, J. S. (2008). A new ghost cell / level set method for moving boundary problems, Application to tumor growth. J. Sci. Comput., 35, 266C299.

Mainardi, F. (1996). Fractional relaxation-oscillation and fractional diffusion-wave phenomena. Chaos. Soliton. Fract., 7(9), 1461C1477.

Meerschaert, M. M., Benson, D. A., Scheffler, H. P., \& BeckerKern, P. (2002). Governing equations and solutions of anomalous random walk limits. Phys. Rev. E., 66, $102 \mathrm{C} 105$.

Meerschaert, M. M., Scheffler, H. P., \& Tadjeran, C. (2006). Finite difference methods for two-dimensional fractional dispersion equation. J. Comput. Phys., 211(1), 249C261.

Meerschaert, M. M., \& Tadjeran, C. (2006). Finite difference approximations for two-sided space-fractional partial differential equations. Appl. Numer. Math., 56, 80C90.

Murio, D. A. (2008). Implicit finite difference approximation for time fractional diffusion equations. Comput. Math. Appl., 56, $1138 \mathrm{C} 1145$.

Mustapha, K. \& McLean, W. (2011). Piecewise-linear, discontinuous galerkin method for a fractional diffusion equation. Numer. Algorithms., 56, 159C184.

Oevermann, M., \& Klein, R. (2006). A cartesian grid finite volume method for elliptic equations with variable coefficients and embedded interfaces. J. Comput. Phys., 219, 749C769.

Oevermann, M., Scharfenberg, C., \& Klein, R. (2009). A sharp interface finite volume method for elliptic equations on cartesian grids. J. Comput. Phys., 228, 5184C5206.

Peskin, C. (1977). Numerical analysis of blood flow in the heart. J. Comput. Phys., 25, 220C252.

Peskin, C., \& Printz, B. (1993). Improved volume conservation in the computation of flows with immersed elastic boundaries. J. Comput. Phys., 105, 33C46.

Ray, S. (2007). Exact solutions for time-fractional diffusion-wave equations by decomposition method. Physica Scripta., $75(1), 53 \mathrm{C} 61$.

Rihan, F. (2010). Computational methods for delay parabolic and time fractional partial differential equations. Numer. Methods Partial Differential Equations, 26(6), 1556C1571.

Roop, J. P. (2006). Computational aspects of fem approximation of fractional. J. Comput. Appl. Math., 193, 243C268. 
Rossikhin, Y. A. \& Shitikova, M. V. (1997). Applications of fractional calculusto dynamic problems of linear and nonlinear hereditary mechanics of solids. Appl. Mech. Rev., 50(1), $15 \mathrm{C} 67$.

Su, L., Wang, W., \& Yang, Z. (2009). Finite difference approximations for the fractional advection-diffusion equation. Phys. Lett. A., 373, 4405C4408.

Sussman, M., Smereka, P., \& Osher, S. (1994). A level set approach for computing solutions to incompressible twophase flow. J. Comput. Phys., 114, 146C154.

Wang, L., Hou, S., \& Shi, L. (2017). An improved non-traditional finite element formulation for solving threedimensional elliptic interface problems. Comput. Math. Appl., 73, 374C384.

Wang, L., Hou, S., \& Shi, L. (2018). A numerical method for solving three-dimensional elliptic interface problems with triple junction points,. Adv. Comput. Math., 44, 175C193.

Wang, L., \& Shi, L. (2015). A numerical method for solving matrix coefficient heat equations with interfaces. Numer. Math. Theor. Meth. Appl., 8(4), 475C495.

Ying, W. J., \& Henriquez, C. S. (2007). A kernel-free boundary integral method for elliptic boundary value problems. J. Comput. Phys., 227(2), 1046C1074.

Yu, S., Zhou, Y., \& Wei, G. W. (2007). Matched interface and boundary (MIB) method for elliptic problems with sharpedged interfaces. J. Comput. Phys., 224, 729C756.

Zayernouri, M., \& Karniadakis, G. E. (2014). Fractional spectral collocation method. SIAM J. Sci. Comput., 36(1), A40-A62.

Zeng, F., Li, C., Liu, F., \& Turner, I. (2015). Numerical algorithms for time-fractional subdiffusion equation with secondorder accuracy. SIAM J. Sci. Comput., 37(1), A55CA78.

Zheng, G., \& Wei, T. (2010). Spectral regularization method for a cauchy problem of the time fractional advectiondispersion equation. J. Comput. Appl. Math., 233(10), 2631C2640.

Zheng, M., Liu, F., Turner, I., \& Anh, V. (2015). A novel high order space-time spectral method for the time fractional fokker-planck equation. SIAM J. Sci. Comput., 37(2), A701CA724.

Zhou, Y. C., Zhao, S., Feig, M., \& Wei, G. W. (2006). High order matched interface and boundary method for elliptic equations with discontinuous coefficients \& singular sources. J. Comput. Phys., 213, 1C30.

\section{Copyrights}

Copyright for this article is retained by the author(s), with first publication rights granted to the journal.

This is an open-access article distributed under the terms and conditions of the Creative Commons Attribution license (http://creativecommons.org/licenses/by/4.0/). 